\title{
COVID-19 und Kopfschmerzen
}

\author{
Pohl, Heiko ; Gantenbein, Andreas R
}

\begin{abstract}
Zusammenfassung. Kopfschmerzen sind ein häufiges Symptom einer COVID-19-Infektion und werden typischerweise als bilateral lokalisiert, frontal betont, drückend und mittelstark bis stark beschrieben. Die Unterscheidung von primären Kopfschmerzerkrankungen gelingt in der Regel durch die systematische Suche nach «Red Flags»; meist werden die Kopfschmerzen von Fieber, Husten oder erhöhten Entzündungsparametern im Blut begleitet. Früher geäusserte Bedenken gegen den Einsatz von Ibuprofen zur symptomatischen Kopfschmerztherapie bei Patientinnen und Patienten mit COVID19 werden durch prospektive Studien nicht unterstützt. Während das Tragen von Masken sehr oft zu Kopfschmerzen führt, die wahrscheinlich durch Kompression sensibler Nerven ausgelöst werden, wirkt sich der Lockdown in der Regel positiv auf eine vorbestehende Migräne aus. Die Behandlung von Betroffenen mit primären Kopfschmerzen wird durch Quarantäneregeln erschwert, weshalb viele Zentren auf virtuelle Konsultationen setzen. COVID-19 and Headaches Abstract. Headaches are a common symptom of COVID-19 infections. Patients generally describe them as bilateral, predominantly frontal, squeezing and of moderate or severe intensity. Searching for "Red Flags" often allows distinction from primary headaches - usually fever, cough, and elevated inflammatory markers accompany COVID-19-associated headaches. Prospective studies did not confirm caveats against the use of ibuprofen as symptomatic treatment. While carrying facial masks often caused headaches, probably by compressing sensory nerves, many patients' migraine frequencies dropped during lockdown. Treatment of patients with primary headaches was complicated by quarantine and many centres offered online consultations. Résumé. Des maux de tête accompagnent souvent les infections au COVID-19. En général, les patients les décrivent comme bilatéraux, frontaux, oppressants et modérés à sévères. En cherchant des «Red Flags», on réussit normalement à les distinguer des maux de têtes primaires. Des études prospectives n'ont pas confirmées la crainte que l'usage de l'ibuprofène pour le traitement de la douleur accompagnant l'infection pourrait compliquer le cours de la maladie. Tandis que porter un masque suscite souvent des maux de tête probablement en comprimant des nerfs sensibles du visage, le confinement réduit souvent la fréquence des crises de migraine. Le traitement des patients souffrants des céphalées primaires a été rendu difficile par le confinement, d'où l'offerte de consultations online par beaucoup de médecins.
\end{abstract}

DOI: https://doi.org/10.1024/1661-8157/a003661

Other titles: COVID-19 and Headaches

Posted at the Zurich Open Repository and Archive, University of Zurich

ZORA URL: https://doi.org/10.5167/uzh-209817

Journal Article

Accepted Version

Originally published at:

Pohl, Heiko; Gantenbein, Andreas R (2021). COVID-19 und Kopfschmerzen. Praxis, 110(4):201-206. 
DOI: https://doi.org/10.1024/1661-8157/a003661 


\section{COVID-19 und Kopfschmerzen}

\section{Englischer Titel \\ COVID-19 and Headache}

\section{Autoren}

Heiko Pohla (ORCID identifier: 0000-0002-2778-6790), Andreas R. Gantenbein ${ }^{a, b}$

${ }^{a}$ Klinik für Neurologie, Universitätsspital Zürich, Schweiz

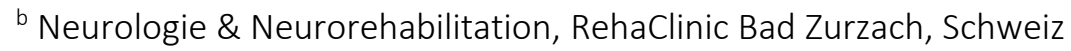

\section{Abkürzungen}

ACE2 Angiotensin-Converting Enzyme 2

FFP filtering face piece

NSAR nicht-steroidale Antirheumatika

SARS Severe acute respiratory syndrome

WHO World Health Organisation, Weltgesundheitsorganisation

\section{Zusammenfassung}

Kopfschmerzen sind ein häufiges Symptom einer COVID-19-Infektion und werden typischerweise als bilateral lokalisiert, frontal betont, drückend und mittelstark bis stark beschrieben. Die Unterscheidung von primären Kopfschmerzerkrankungen gelingt in der Regel durch die systematische Suche nach «Red Flags»; meist werden die Kopfschmerzen von Fieber, Husten oder erhöhten Entzündungsparametern im Blut begleitet. Früher geäusserte Bedenken gegen den Einsatz von Ibuprofen zur symptomatischen Kopfschmerztherapie bei Patienten mit COVID-19 werden durch prospektive Studien nicht unterstützt.

Während das Tragen von Masken sehr oft zu Kopfschmerzen führt, die wahrscheinlich durch Kompression sensibler Nerven ausgelöst werden, wirkt sich der Lockdown in der Regel positiv auf eine vorbestehende Migräne aus. Die Behandlung von Patienten mit primären Kopfschmerzen wird durch Quarantäneregeln erschwert, weshalb viele Zentren auf virtuelle Konsultationen setzten.

\section{Summary}

Headaches are a common symptom of COVID-19 infections. Patients generally describe them as bilateral, predominantly frontal, squeezing and of moderate or severe intensity. Searching for "Red Flags" often allows distinction from primary headaches - usually fever, cough, and elevated inflammatory markers accompany COVID-19 associated headaches. Prospective studies did not confirm caveats against the use of ibuprofen as symptomatic treatment.

While carrying facial masks often caused headaches in healthcare professionals, probably by compressing sensory nerves, many patients' migraine frequencies dropped during lockdown. Treatment 
of patients with primary headaches was complicated by quarantine and many centres offered online consultations.

\section{Résumé}

Des maux de tête accompagnent souvent les infections au COVID-19. En général, les patients les décrivent comme bilatéraux, frontaux, oppressants et modérés à sévères. En cherchant des "Red Flags », on réussit normalement à les distinguer des maux de têtes primaires. Des études prospectives n'ont pas confirmées la crainte que l'usage de l'ibuprofène pour le traitement de la douleur accompagnant l'infection pourrait compliquer le cours de la maladie.

Tandis que porter un masque suscite souvent des maux tête probablement en comprimant des nerfs sensibles du visage, le confinement réduit souvent la fréquence des crises de migraine. Le traitement des patients souffrants des céphalées primaires a été rendu difficile par le confinement d'où l'offerte de consultations online par beaucoup de médecins.

\section{Schlüsselwörter}

Deutsch: SARS-COV2; Maske; Migräne; Quarantäne; Lockdown

English: SARS-COV2; mask; migraine; quarantine; Lockdown

Français: SARS-COV2; masque; migraine; quarantaine; confinement 


\section{Einleitung}

Die Symptome des ersten nachweislich mit COVID-19 infizierten Patienten traten im Dezember 2019 auf.[1] Das Virus breitete sich in den folgenden Wochen rasch weltweit aus. In der Schweiz wurde der erste Fall am 25.02.2020 bestätigt.[2] Nachdem die Weltgesundheitsorganisation WHO am 30.01.2020 den internationalen Gesundheitsnotstand («Public Health Emergency of International Concern») erklärt hatte, sprach sie ab dem 11.03.2020 von einer Pandemie.[3, 4]

Wie schon bei früheren Pandemien, [5] gehören Kopfschmerzen auch bei der COVID-19-Infektion zu den häufigen Symptome.[6, 7] Da diese aber auch unabhängig von viralen Infekten sehr häufig sind,[8] war deren diagnostischer Wert zunächst unklar. Aus diesem Grund versuchten zahlreiche Forschungsgruppen ihren Phänotyp zu charakterisieren und Unterschiede zu anderen Kopfschmerztypen herauszustellen.[9-12]

Um die Ausbreitung des Virus einzudämmen wurden Massnahmen getroffen, die weitreichende Auswirkungen auf das Leben fast aller Menschen hatten. Da Migräneattacken möglicherweise oft durch Umweltreize ausgelöst werden,[13] stellte sich deshalb die Frage, wie deren Häufigkeit durch die Pandemie beeinflusst $[14,15]$ und wie sich die Kopfschmerzbehandlung selbst verändern würde.[16-19]

Das Ziel dieses Mini-Reviews ist es, den Kenntnisstand über die Kopfschmerzen infolge einer COVID-19Infektion und die Auswirkungen der Pandemie auf Kopfschmerzen und deren Behandlung zusammenzufassen.

\section{Kopfschmerzen als Symptom einer COVID-19-Infektion}

Eine COVID-19-Infektion geht oft mit Kopfschmerzen einher.[6] Studien berichten eine Häufigkeit zwischen 7 und 75\%.[1, 20-25] Interessanterweise liegen die Zahlen in asiatischen Ländern zwischen 7\% und $14 \%,[1,24,25]$ und somit niedriger als in Europa, wo Kopfschmerzen bei $24 \%$ bis $75 \%[20,21,23]$ dokumentiert wurden. Nachdem schon länger bekannt war, dass die Prävalenz primärer Kopfschmerzen in China niedriger ist,[8] scheint sich die Anfälligkeit auch für sekundäre Kopfschmerzen in ähnlicher Weise zu unterscheiden.

Mehrere Studien - nicht alle[11, 26] - zeigen zudem, dass Frauen mit einer COVID-19-Infektion häufiger Kopfschmerzen haben als Männer.[9, 20, 21, 23] Somit scheint sich auch hier das von den meisten primären Kopfschmerzen gewohnte Bild zu bestätigen.[8]

Der Kopfschmerzphänotyp wird häufig als bilateral, frontal betont, drückend und mittelstark bis stark beschrieben.[9-11] Zudem hatten etwas mehr als die Hälfte der Patienten eine Überempfindlichkeit auf sensorische Reize und somit einen eher migräneartigen Phänotyp.[9, 11]

Die Schwere der COVID-19-Infektion scheint zwar nicht im Zusammenhang mit der An- oder Abwesenheit der Kopfschmerzen zu stehen.[27] Es gibt aber Hinweise darauf, dass Kopfschmerzen mit einem kürzeren Verlauf der Infektionserkrankung verbunden sein könnten.[20] Sie treten bei ca. einem Viertel der Patienten als Erstsymptom auf[9] und sistieren bei ca. zwei Drittel der Patienten spontan wieder; bei ca. einem Drittel der Patienten persistierten sie jedoch auch noch nach mehreren Wochen (s.u.).[20]

Kopfschmerzen, die während einer viralen Infektion auftreten, werden als «Akuter Kopfschmerz zurückzuführen auf eine systemische virale Infektion [ICHD-3 9.2.2.1]» klassifiziert. Bestehen die Kopfschmerzen insgesamt länger als drei Monate, die Abheilung der Infektion selbst liegt aber weniger als drei Monate zurück, liegt ein «Chronischer Kopfschmerz zurückzuführen auf eine systemische virale Infektion [ICHD-3 9.2.2.2]» vor. 
Inzwischen werden Kopfschmerzen als ein auf eine COVID19-Erkrankung hinweisendes Symptom interpretiert.[30] Da aber Kopfschmerzen auch bei Patienten ohne Infektion häufig sind,[8] ist der positiv prädiktive Wert dieses Symptoms alleine zu gering.[31] Eine begleitende Anosmie oder Ageusie deuten zwar mit hoher Sensitivität auf einen sekundären Kopfschmerzen hin, traten aber nur bei rund einem Drittel aller Patienten mit COVID-19-assoziierten Kopfschmerzen auf.[11] Eine Studie untersuchte daher, wie gut es im klinischen Alltag gelingt, COVID-19-assoziierte Kopfschmerzen von primären Kopfschmerzen zu unterscheiden. [12] Garcia-Azorin et al. stellten hierbei fest, dass Verwechslungen mit primären Kopfschmerzen in den meisten Fällen unwahrscheinlich sind. Voraussetzung ist aber, dass man systematisch nach «Red Flags» sucht, also Krankheitszeichen, die auf einen sekundären Kopfschmerz hinweisen.[32] In ihrer Studie wurden bei allen 104 Patienten mit bestätigter COVID-19-Infektion und Kopfschmerzen «Red Flags» gefunden - am häufigsten waren Fieber, Husten oder erhöhte Entzündungsparameter im Blut.[12]

\section{Pathophysiologie der mit COVID-19-assoziierten Kopfschmerzen}

Die Pathophysiologie der COVID-19-assoziierten Kopfschmerzen ist nicht bekannt. Eine virale Meningitis ist unwahrscheinlich, bedenkt man, dass das Virus in einer Fallserie bei Patienten mit Kopfschmerzen im Liquor nicht nachgewiesen wurde.[33] Wahrscheinlicher ist das Vorliegen eines «Kopfschmerz zurückzuführen auf eine systemische virale Infektion».[28]

Eine Hypothese ist, dass Endäste des Nervus trigeminus direkt durch das Virus infiltriert und dadurch aktiviert werden könnten. Hiergegen spricht aber, dass Rezeptoren des Angiotensin-Converting Enzyme 2 (ACE2), welches für die Infiltration essentiell zu sein scheint, in den trigeminalen Nervenendigungen nicht nachgewiesen wurden.[34] Da ACE2 aber im Endothel exprimiert wird, könnte das Virus dort eindringen und so den Trigeminusnerv reizen. Schliesslich könnten auch im Blut zirkulierende Entzündungsmediatoren zur Entstehung der Schmerzen beitragen.[34]

Da Kopfschmerzen häufig mit Anosmie und Ageusie einhergehen, wird auch ein Eindringen des Virus in das zentrale Nervensystem über das Riechepithel diskutiert.[11, 35] Coppola et al. vermuten zudem, dass die Ursache der Schmerzen eher im mit der Erkrankung assoziierten Stress liegen könnte, statt in den Viren selbst.[36]

Einzelne Fallberichte und eine Fallserie deuten darauf hin, dass die Kopfschmerzen in manchen Fällen auch durch eine intrakranielle Drucksteigerung ausgelöst wurden.[33, 37-39] Interessanterweise waren Frauen hiervon häufiger betroffen - ähnlich wie bei der idiopathischen intrakraniellen Drucksteigerung.[33, 40, 41]

Die Kopfschmerzen der Patienten mit intrakranieller Drucksteigerung wurden als holozephal und pulsierend beschrieben und bestanden jeden Tag.[33] Eine Lageabhängigkeit der Schmerzen, die als „Red Flag" auf eine intrakrankielle Drucksteigerung hindeuten könnte,[32] wurde nur in einem Fall berichtet.[38] Eine Papillenschwellung bestand ebenfalls nicht in allen Fällen, sodass unklar ist, ob die Drucksteigerung immer mit der Gefahr der Erblindung einhergeht.[33] Die kleinen Fallzahlen erschweren die Interpretation der Daten zudem. Die Kopfschmerzen besserten sich bei den meisten Patienten mit intrakranieller Drucksteigerung innerhalb weniger Tage.[33] Es ist somit möglich, dass sich auch der Druck rasch wieder normalisiert. Eine definitive Aussage ist noch nicht möglich.

Keine der zitierten Publikationen berichtet Prävalenzdaten zur intrakraniellen Drucksteigerung bei COVID-19-Patienten. Zudem scheint sich der berichtete Phänotyp von anderen Kopfschmerztypen nicht deutlich zu unterscheiden, sodass aktuell nicht klar ist, wie betroffene Patienten identifiziert werden könnten und wie häufig die Erkrankung ist. Bis zur Sammlung weiterer klinischer Daten empfehlen wir, 
die Indikation für weiterführende Untersuchungen grosszügig zu stellen, wenn Patienten über Sehprobleme berichten.

\section{Behandlung COVID-19-assoziierter Kopfschmerzen}

Bei akuten Kopfschmerzen werden häufig nicht-steroidale Antirheumatika (NSAR) eingesetzt. Da aus einem Tierexperiment abgeleitet wurde, dass Ibuprofen möglicherweise die Expression von ACE2Rezeptoren steigert und COVID-19 über diese in Wirtszellen eindringt, wurde ein schwererer Krankheitsverlauf durch Einsatz dieser Medikamente befürchtet.[42-44] Unbekannt war jedoch, ob es diesen Effekt beim Menschen gibt und er im klinischen Alltag eine Rolle spielen würde. Dementsprechend betonten Medikamentenbehörden und die Therapiekommission der Schweizerischen Kopfwehgesellschaft, dass aufgrund der Datenlage ein Verzicht auf diese Medikamente nicht empfohlen werden kann. [45-48] Inzwischen bestätigten zwei prospektive Studien, dass ihr Einsatz den Verlauf einer COVID-19-Infektion nicht negativ beeinflusst.[49, 50]

Bei längerfristig bestehenden Kopfschmerzen sollten nicht nur Akutmedikamente, sondern auch medikamentöse Prophylaxen eingesetzt werden. Welches Medikament bei bleibenden Kopfschmerzen nach einer COVID-19-Infektion nützlich sein könnte, wurde bislang nicht untersucht. Es gibt Erfahrungen aus sehr kleinen Stichproben auf die zurückgegriffen werden kann.

Prakash und Shah berichteten, dass alle neun Patienten, die wegen persistierender post-infektiöser Kopfschmerzen mit Methylprednisolon behandelt wurden, eine Besserung berichteten.[51] Andere Empfehlungen beinhalten eine Behandlung mit Doxycyclin, Nervenblockaden mit Bupivacain, BotoxInjektionen, intravenöse Gabe von Lidocain und Ketamin sowie Nimodipin.[52]

\section{Langzeitfolgen einer COVID-19-Erkrankung}

Nicht nur Kopfschmerzen können als Residualsymptom nach einer COVID-19-Infektion persistieren. Die WHO berichtete Ende Oktober 2020, dass einige Beschwerden auch noch zwei Wochen nach der Infektion bestehen können.[53,54] Ähnliche Erfahrungen waren bereits nach der SARS-Pandemie von 2003 gemacht worden.[55, 56]

Die persistierenden Symptome sind häufig unspezifisch, wie Kopfschmerzen, Müdigkeit, Konzentrationsstörungen und die Frage der Kausalität ist noch nicht geklärt. Um ähnlichen, vorwiegend versicherungsmedizinischen Schwierigkeiten, wie sie z.B. bei der Borreliose oder bei posttraumatischen Kopfschmerzen bestehen, vorzubeugen, sollten unbedingt Kohorten prospektiv begleitet und Risikofaktoren für die Chronifizierung erkannt werden.

\section{Auswirkungen von Pandemie und Quarantäne auf Kopfschmerzen}

Die rasche Verbreitung des unsichtbaren Virus, die grundsätzlichen Schwierigkeiten asymptomatische Patienten zu identifizieren und die v.a. zu Beginn der Pandemie geringe Anzahl zur Verfügung stehender Tests waren und bleiben grosse Herausforderungen der COVID-19-Pandemie. Um Ansteckungen zur verhindern, wurden daher in vielen Ländern Schutzmassnahmen eingeführt (Quarantäne, Mobilitätseinschränkungen, «lock down»).

Auch wenn sich Regeln zur Isolation im Krankheitsfall bereits in der Thora bzw. dem Alten Testament (3. Mose 13,4 ff.) finden, wurde die erste institutionalisierte Quarantäne erst im 14. Jahrhundert verhängt. Damals wurden zur Eindämmung der Pestepidemie potentiell infizierte Personen zunächst in Dubrovnik, Kroatien, später in Venedig, Italien und schliesslich auch in vielen anderen Ländern von der restlichen Bevölkerung isoliert. Dabei deutet der Name Quarantäne (von ital. quaranta - vierzig) die Dauer der 
Ausgangssperre von 40 Tagen an. Auch wurde in Venedig schon in dieser Zeit ein Sicherheitsabstand («social distancing») bei der Kommunikation mit den Kapitänen einlaufender Frachtschiffe eingehalten.[57]

Konzeptuell bedeutet das Verhängen solcher Schutzmassnahmen, dass der Krankheitseindämmung höhere Priorität beigemessen wird, als wirtschaftlichen Interessen, Reisefreiheit und Bedürfnissen nach sozialer Interaktion. So überrascht es nicht, dass Menschen, die sich in der Vergangenheit in Quarantäne begeben mussten, neben Angst vor Ansteckung auch über finanzielle Sorgen, Zukunftsängste und Einsamkeit sowie einen veränderten Tag-Nacht-Rhythmus berichteten. $[58,59]$

Die Auswirkung einer Quarantäne auf Migränepatienten war vor Beginn der COVID-19-Pandemie noch nie untersucht worden. Da aber viele Patienten Stress, Ängste oder unregelmässigen Schlaf als Auslöser ihrer Migräneanfälle erleben,[13] war zu befürchten, dass die Quarantäne zu einer Zunahme der Migränetage führen könnte.[60] Zwei inzwischen publizierte Studien bestätigen diese Sorge aber nicht.[14, 15]

Delussi et al. führten ungefähr einen Monat nach Beginn der Quarantänemassnahmen Telefoninterviews mit 433 Migränepatienten durch, von denen die meisten unter einer episodischen Migräne litten. Sie stellten fest, dass sich die Migräne im Allgemeinen leicht gebessert hatte und das Ausmass der Besserung mit der Anzahl zu Hause verbrachter Tage korrelierte. Es zeigte sich auch, dass die Migränehäufigkeit nicht abnahm, wenn Patienten Angst hatten sich anzustecken.[14]

Eine ähnliche Untersuchung führten Papetti et al. in einem pädiatrischen Sample durch und baten 707 Kinder und Jugendliche zwischen fünf und 18 Jahren während des «Lockdown» um das Ausfüllen eines Online-Fragebogens. Knapp die Hälfte der Befragten (46\%) berichtete über eine Verbesserung, während 15\% eine Verschlechterung ihrer Kopfschmerzerkrankung im Allgemeinen angaben. Die Anzahl der Kopfschmerztage sank von durchschnittlich 7.38 Tage vor dem Lockdown auf 5.4 Tage. Am deutlichsten verbesserte sich die Migräne besonders schwer betroffener Patienten.[15]

Die Konzentration personeller Ressourcen im Gesundheitssektor auf die Eindämmung der Pandemie führte zu Versorgungslücken an anderer Stelle. Vielerorts war eine persönliche Konsultation des Neurologen zur Kopfschmerzbehandlung nicht mehr möglich; stationäre Therapien wurden abgesagt und z.B. Injektionen mit Botulinum-Toxin konnten oftmals nicht erfolgen. Obwohl Zuweisungen neuer Patienten zurückgingen, wuchsen Wartelisten. In vielen Spitälern wich man auf Telefonkonsultationen oder Videokonferenzen aus, um den Bedürfnissen der Patienten bestmöglich gerecht zu werden.[16$19,61]$

\section{Kopfschmerzen als Folge des Tragens einer Gesichtsmaske}

Nach anfänglichem Zögern wurde das Tragen einer Mund-Nasen-Maske, häufig verbunden mit dem Tragen einer Schutzbrille, fester Bestandteil der Strategie zur Krankheitseindämmung in den Spitälern. Obwohl das Tragen einer FFP2-Maske mit einem signifikanten $\mathrm{CO}_{2}$-Anstieg einhergeht, kommt es nicht zu Hyperkapnie oder Hypoxie.[62] Sehr viele Menschen berichten aber über durch das Tragen einer Maske ausgelöste Kopfschmerzen. Dieses Phänomen war bereits aus der Vergangenheit bekannt und wurde im Zusammenhang mit COVID-19 in zwei Studien untersucht.[63, 64]

Ong et al. befragten 158 Mitarbeiter eines Spitals, die während der Arbeitszeit Gesichtsmasken und Schutzbrillen trugen. Neuartige Kopfschmerzen wurden dabei von der grossen Mehrheit aller Befragten (81.1\%) berichtet - unabhängig davon, ob zusätzlich Schutzbrillen getragen wurden. Die Schmerzen betrafen dabei in erster Linie die Stellen, die durch Maske, Schutzbrille oder Gummibänder komprimiert wurden. Bei den meisten Betroffenen traten die Schmerzen sehr schnell auf, meist innerhalb von weniger als 60 Minuten, und verschwanden nach Absetzen der Schutzausrüstung auch wieder schnell, 
in der Regel innerhalb von 30 Minuten. Die wichtigsten Risikofaktoren für das Auftreten von Kopfschmerzen waren vorbestehende primäre Kopfschmerzen und das Tragen der Maske während mehr als vier Stunden pro Tag.[63]

Ramirez-Moreno et al. untersuchten 306 Mitarbeiter des Gesundheitssystems und berichteten über neuartige Kopfschmerzen bei etwas mehr als der Hälfte der Befragten.[64] Sie waren häufiger bei Frauen, Pflegekräften (verglichen mit dem ärztlichen Personal und «anderen») und der Verwendung von FFP2-Masken (verglichen mit chirurgischen Masken).

Da die Lokalisation der Kopfschmerzen mit den Orten der Kompression durch die Schutzausrüstung übereinstimmt, [63] ist der Kopfschmerz am ehesten als «Kopfschmerz durch Einwirkung von Druck oder Zug auf den Kopf» zu klassifizieren. Interessanterweise handelt es sich dabei - gemäss der dritten Auflage der Internationalen Kopfschmerzklassifikation - um einen primären Kopfschmerz.[28] Dieser Kopfschmerztyp war in der zweiten Auflage in die Klassifikation aufgenommen, damals aber noch nicht als primär, sondern als Neuralgie klassifiziert worden.[65]

Unklar ist, ob auch der Anstieg des $\mathrm{CO}_{2}$-Partialdrucks zum Auftreten der Kopfschmerzen beitragen könnte, auch wenn das Tragen einer Maske nicht zu Hyperkapnie führt.[64, 66]

\section{Ausblick}

Die COVID-19-Pandemie weckte erhebliches Forschungsinteresse, das zu einem rasanten Wissensanstieg führte. Besonders wichtig erscheint uns gerade auch im Hinblick auf die Zeit nach der Quarantäne, dass sich eine Migräneerkrankung durch eine flexible Alltagsgestaltung und Verringerung der Verpflichtungen positiv beeinflussen lässt.[63, 64] Auch wenn die gute Wirkung der nichtmedikamentösen Migränebehandlung schon länger bekannt ist,[67] haben Home-Office und Gleitzeit noch keinen Eingang in die Therapieempfehlungen gefunden. Ideal wäre, wenn die Ausrichtung des Lebensstils an den eigenen Bedürfnissen nach dem Ende der Pandemie selbstverständlich würde.

\section{Key messages}

- Kopfschmerzen treten häufig im Rahmen einer COVID-19-Infektion auf und werden typischerweise als bilateral lokalisiert, frontal betont, drückend und mittelstark bis stark beschrieben.

- Migränepatienten berichteten im Allgemeinen eine Besserung ihrer Kopfschmerzen während Quarantäne und Lockdown.

- Das Tragen einer Gesichtsmaske führt bei vielen Menschen zu Kopfschmerzen, die wahrscheinlich in erster Linie durch Kompression sensibler Gesichtsnerven ausgelöst werden.

\section{Lernfragen}

Wie hat der vielerorts verhängte Lock-Down die Anfallsfrequenz bei Menschen mit Migräne im Allgemeinen beeinflusst?
a. Kein Einfluss
b. Es kam zu einer Zunahme der Migränetage
c. Es kam zu einer Abnahme der Migränetage

Antwort: C 
Welches ist die wahrscheinlichste Ursache der durch das Tragen einer FFP2-Maske ausgelösten Kopfschmerzen?
a. Hyperkapnie
b. Druckkompression sensibler Nerven
c. Es handelt sich um psychosomatische Schmerzen
d. Hypoxie

Antwort: B

\section{Interessenskonflikt}

Die Autoren erklären, dass kein Interessenskonflikt vorliegt.

\section{Literaturangaben}

1. Huang, C., et al., Clinical features of patients infected with 2019 novel coronavirus in Wuhan, China. The Lancet, 2020. 395(10223): p. 497-506.

2. Bundesamt für Gesundheit. Neues Coronavirus COVID-19: Erster bestätigter Fall in der Schweiz 2020 [cited 2021 13/01/2021]; Available from: https://www.bag.admin.ch/bag/de/home/dasbag/aktuell/medienmitteilungen.msg-id-78233.html.

3. World Health Organization. WHO Director-General's opening remarks at the media briefing on COVID-19 - 11 March 2020. 2020 [cited 2021 09/01/2020]; Available from: https://www.who.int/director-general/speeches/detail/who-director-general-s-openingremarks-at-the-media-briefing-on-covid-19---11-march-2020.

4. World Health Organization. Statement on the second meeting of the International Health Regulations (2005) Emergency Committee regarding the outbreak of novel coronavirus (2019nCoV). 2020 [cited 2021 09/01/2021]; Available from: https://www.who.int/news/item/30-012020-statement-on-the-second-meeting-of-the-international-health-regulations-(2005)emergency-committee-regarding-the-outbreak-of-novel-coronavirus-(2019-ncov).

5. Rozen, T.D., Daily persistent headache after a viral illness during a worldwide pandemic may not be a new occurrence: Lessons from the 1890 Russian/Asiatic flu. Cephalalgia, 2020. 40(13): p. 1406-1409.

6. Lippi, G., et al., Headache is an important symptom in patients with coronavirus disease 2019 (COVID-19). Diagnosis (Berl), 2020. 7(4): p. 409-411.

7. Islam, M.A., et al., Prevalence of Headache in Patients With Coronavirus Disease 2019 (COVID19): A Systematic Review and Meta-Analysis of 14,275 Patients. Front Neurol, 2020. 11: p. 562634.

8. Stovner, L.J., et al., Global, regional, and national burden of migraine and tension-type headache, 1990-2016: a systematic analysis for the Global Burden of Disease Study 2016. The Lancet Neurology, 2018. 17(11): p. 954-976.

9. Lopez, J.T., et al., Phenotypic characterization of acute headache attributed to SARS-CoV-2: An ICHD-3 validation study on 106 hospitalized patients. Cephalalgia, 2020. 40(13): p. 1432-1442.

10. Magdy, R., et al., Characteristics of headache attributed to COVID-19 infection and predictors of its frequency and intensity: A cross sectional study. Cephalalgia, 2020. 40(13): p. 1422-1431.

11. Rocha-Filho, P.A.S. and J.E. Magalhaes, Headache associated with COVID-19: Frequency, characteristics and association with anosmia and ageusia. Cephalalgia, 2020. 40(13): p. 14431451.

12. Garcia-Azorin, D., et al., Frequency and Type of Red Flags in Patients With Covid-19 and Headache: A Series of 104 Hospitalized Patients. Headache, 2020. 60(8): p. 1664-1672.

13. Park, J.W., et al., Analysis of Trigger Factors in Episodic Migraineurs Using a Smartphone Headache Diary Applications. PLoS One, 2016. 11(2): p. e0149577. 
14. Delussi, M., et al., Investigating the Effects of COVID-19 Quarantine in Migraine: An Observational Cross-Sectional Study From the Italian National Headache Registry (RICe). Front Neurol, 2020. 11: p. 597881.

15. Papetti, L., et al., I stay at home with headache. A survey to investigate how the lockdown for COVID-19 impacted on headache in Italian children. Cephalalgia, 2020. 40(13): p. 1459-1473.

16. Kristoffersen, E.S., et al., Hospital-based headache care during the Covid-19 pandemic in Denmark and Norway. J Headache Pain, 2020. 21(1): p. 128.

17. Lopez-Bravo, A., et al., Impact of the COVID-19 pandemic on headache management in Spain: an analysis of the current situation and future perspectives. Neurologia, 2020. 35(6): p. 372-380.

18. Gatti, F. and L. Manneschi, Headache relevance in outpatient activity during Covid-19 pandemic. Neurol Sci, 2020. 41(Suppl 2): p. 481-482.

19. Grazzi, L. and P. Rizzoli, The Adaptation of Management of Chronic Migraine Patients With Medication Overuse to the Suspension of Treatment Protocols During the COVID-19 Pandemic: Lessons From a Tertiary Headache Center in Milan, Italy. Headache, 2020. 60(7): p. 1463-1464.

20. Caronna, E., et al., Headache: A striking prodromal and persistent symptom, predictive of COVID19 clinical evolution. Cephalalgia, 2020. 40(13): p. 1410-1421.

21. Trigo, J., et al., Factors associated with the presence of headache in hospitalized COVID-19 patients and impact on prognosis: a retrospective cohort study. J Headache Pain, 2020. 21(1): p. 94.

22. Peng, K.P., Association between COVID-19 and headache: What evidence and history tell us. Cephalalgia, 2020. 40(13): p. 1403-1405.

23. Lechien, J.R., et al., Clinical and epidemiological characteristics of 1420 European patients with mild-to-moderate coronavirus disease 2019. J Intern Med, 2020. 288(3): p. 335-344.

24. Guan, W.J., et al., Clinical Characteristics of Coronavirus Disease 2019 in China. N Engl J Med, 2020. 382(18): p. 1708-1720.

25. Tian, S., et al., Characteristics of COVID-19 infection in Beijing. J Infect, 2020. 80(4): p. 401-406.

26. Uygun, O., et al., Headache characteristics in COVID-19 pandemic-a survey study. J Headache Pain, 2020. 21(1): p. 121.

27. Ramphul, K., S.G. Mejias, and Y. Ramphul, Headache may not be linked with severity of coronavirus disease 2019 (COVID-19). World J Emerg Med, 2020. 11(4): p. 274.

28. Headache Classification Committee of the International Headache Society (IHS) The International Classification of Headache Disorders, 3rd edition. Cephalalgia, 2018. 38(1): p. 1211.

29. Rozen, T.D., Triggering Events and New Daily Persistent Headache: Age and Gender Differences and Insights on Pathogenesis-A Clinic-Based Study. Headache, 2016. 56(1): p. 164-73.

30. Bundesamt für Gesundheit. Coronavirus: Krankheit, Symptome, Behandlung. 2021 [cited 2021 10/01/2021]; Available from: https://www.bag.admin.ch/bag/de/home/krankheiten/ausbrueche-epidemienpandemien/aktuelle-ausbrueche-epidemien/novel-cov/krankheit-symptome-behandlungursprung.html.

31. Pohl, H., et al., Green Flags and headache: A concept study using the Delphi method. Headache, 2021.

32. Do, T.P., et al., Red and orange flags for secondary headaches in clinical practice: SNNOOP10 list. Neurology, 2019. 92(3): p. 134-144.

33. Silva, M.T.T., et al., Isolated intracranial hypertension associated with COVID-19. Cephalalgia, 2020. 40(13): p. 1452-1458.

34. Bolay, H., A. Gul, and B. Baykan, COVID-19 is a Real Headache! Headache, 2020. 60(7): p. 14151421.

35. Sampaio Rocha-Filho, P.A. and L. Voss, Persistent Headache and Persistent Anosmia Associated With COVID-19. Headache, 2020. 60(8): p. 1797-1799.

36. Coppola, A., et al., Headache in a group of SARS-COVID-19 patients: an observational prospectical study. Neurol Sci, 2020. 41(Suppl 2): p. 503-504. 
37. Noro, F., F.M. Cardoso, and E. Marchiori, COVID-19 and benign intracranial hypertension: A case report. Rev Soc Bras Med Trop, 2020. 53: p. e20200325.

38. Seth, V. and S. Kushwaha, Headache Due to COVID-19: A Disabling Combination. Headache, 2020. 60(10): p. 2618-2621.

39. Svedung Wettervik, T., et al., Intracranial Pressure Dynamics and Cerebral Vasomotor Reactivity in Coronavirus Disease 2019 Patient With Acute Encephalitis. Crit Care Explor, 2020. 2(8): p. e0197.

40. Kesler, A. and N. Gadoth, Epidemiology of idiopathic intracranial hypertension in Israel. J Neuroophthalmol, 2001. 21(1): p. 12-4.

41. Radhakrishnan, K., et al., Idiopathic intracranial hypertension (pseudotumor cerebri). Descriptive epidemiology in Rochester, Minn, 1976 to 1990. Arch Neurol, 1993. 50(1): p. 78-80.

42. Li, W., et al., Angiotensin-converting enzyme 2 is a functional receptor for the SARS coronavirus. Nature, 2003. 426(6965): p. 450-4.

43. Qiao, W., et al., Ibuprofen attenuates cardiac fibrosis in streptozotocin-induced diabetic rats. Cardiology, 2015. 131(2): p. 97-106.

44. MaassenVanDenBrink, A., T. de Vries, and A.H.J. Danser, Headache medication and the COVID19 pandemic. J Headache Pain, 2020. 21(1): p. 38.

45. European Medicines Agency. EMA gives advice on the use of non-steroidal anti-inflammatories for COVID-19. 2020 18/03/2020; Available from: https://www.ema.europa.eu/en/news/emagives-advice-use-non-steroidal-anti-inflammatories-covid-19.

46. Day, M., Covid-19: European drugs agency to review safety of ibuprofen. BMJ, 2020. 368: p. m1168.

47. U.S. Food and Drug Administration. FDA advises patients on use of non-steroidal antiinflammatory drugs (NSAIDs) for COVID-19. 2020 19/3/2020 [cited 2021 10/01/2021]; Available from: https://www.fda.gov/drugs/drug-safety-and-availability/fda-advises-patients-use-nonsteroidal-anti-inflammatory-drugs-nsaids-covid-19.

48. Schweizerische Kopfwehgesellschagft. Informationen zu COVID-19 und Migräne oder andere Kopfschmerzen. $2020 \quad$ [cited 2021 13/01/2021]; Available from: https://headache.ch/download/Content_attachments/FileBaseDoc/IMSKAD_COVID-19.pdf.

49. Abu Esba, L.C., et al., Ibuprofen and NSAID Use in COVID-19 Infected Patients Is Not Associated with Worse Outcomes: A Prospective Cohort Study. Infect Dis Ther, 2020.

50. Bruce, E., et al., Prior Routine Use of Non-Steroidal Anti-Inflammatory Drugs (NSAIDs) and Important Outcomes in Hospitalised Patients with COVID-19. J Clin Med, 2020. 9(8).

51. Prakash, S. and N.D. Shah, Post-infectious new daily persistent headache may respond to intravenous methy/prednisolone. J Headache Pain, 2010. 11(1): p. 59-66.

52. Yamani, N. and J. Olesen, New daily persistent headache: a systematic review on an enigmatic disorder. J Headache Pain, 2019. 20(1): p. 80.

53. World Health Organization. What we know about long-term effects of COVID-19. 2020 [cited 2021 13/01/2021]; Available from: https://www.who.int/docs/defaultsource/coronaviruse/risk-comms-updates/update-36-long-term-

symptoms.pdf?sfvrsn=5d3789a6_2.

54. Centers for Disease Control and Prevention. Symptom Duration and Risk Factors for Delayed Return to Usual Health Among Outpatients with COVID-19 in a Multistate Health Care Systems Network. $2020 \quad$ [cited 2021 13/01/2021]; Available from: https://www.cdc.gov/mmwr/volumes/69/wr/mm6930e1.htm\#.

55. Ngai, J.C., et al., The long-term impact of severe acute respiratory syndrome on pulmonary function, exercise capacity and health status. Respirology, 2010. 15(3): p. 543-50.

56. Lam, M.H., et al., Mental morbidities and chronic fatigue in severe acute respiratory syndrome survivors: long-term follow-up. Arch Intern Med, 2009. 169(22): p. 2142-7.

57. Tognotti, E., Lessons from the history of quarantine, from plague to influenza A. Emerg Infect Dis, 2013. 19(2): p. 254-9.

58. Brooks, S.K., et al., The psychological impact of quarantine and how to reduce it: rapid review of the evidence. The Lancet, 2020. 395(10227): p. 912-920. 
59. Blume, C., M.H. Schmidt, and C. Cajochen, Effects of the COVID-19 lockdown on human sleep and rest-activity rhythms. Curr Biol, 2020. 30(14): p. R795-R797.

60. Dresler, T., et al., [Psychological treatment of headache in times of COVID-19]. Schmerz, 2020. 34(6): p. 503-510.

61. Szperka, C.L., et al., Migraine Care in the Era of COVID-19: Clinical Pearls and Plea to Insurers. Headache, 2020. 60(5): p. 833-842.

62. Rebmann, T., R. Carrico, and J. Wang, Physiologic and other effects and compliance with longterm respirator use among medical intensive care unit nurses. Am J Infect Control, 2013. 41(12): p. 1218-23.

63. Ong, J.J.Y., et al., Headaches Associated With Personal Protective Equipment - A Cross-Sectional Study Among Frontline Healthcare Workers During COVID-19. Headache, 2020. 60(5): p. 864877.

64. Ramirez-Moreno, J.M., et al., Mask-associated 'de novo' headache in healthcare workers during the COVID-19 pandemic. Occup Environ Med, 2020.

65. ICHD-II Classification: Parts 1-3: Primary, Secondary and Other. Cephalalgia, 2016. 24(1_suppl): p. 23-136.

66. Law, J., et al., Carbon Dioxide Physiological Training at NASA. Aerosp Med Hum Perform, 2017. 88(10): p. 897-902.

67. Goslin, R.E., et al., Behavioral and Physical Treatments for Migraine Headache. 1999, Rockville (MD): Agency for Health Care Policy and Research (US). 